\title{
Loss of core fucosylation influenced c-Met-mediated cell proliferation in HepG2 cells
}

\author{
Yuqin Wang, Yuan Xue, Wei Zhang \\ Department of Pharmacology, Pharmacy College, Nantong University, China
}

$\mathrm{N}$-Linked glycosylation is the most common type of glycosidic bond. It is thought to be important for the folding, the stability, and the vast degree of biological functions of glycoproteins. FUT8 is the only key enzyme that catalyzes a fucose from guanosine diphosphate-fucose (GDP-Fuc) to the innermost N-acetylglucosamine (GlcNAc) residue via a1, 6linkage in mammals. We have reported that loss of FUT8 and core fucosylation inhibited liver regeneration after partial hepatectomy in mice. It was speculated that FUT8 might regulate the biological functions of c-Met, the receptor of hepatocyte growth factor (HGF), which played important roles during liver regeneration. In this study, we constructed the FUT8 gene knockout (KO) HepG2 cell line, and found that the cell proliferation induced by HGF was inhibited in FUT8 KO HepG2 cells. Consistently, the expression of cyclin D1 and E1 were also suppressed in these cells. Furthermore, the treatments with HGF significantly increased the phosphorylation levels of c-Met (Tyr1234/5 and Tyr1349), ERK, and AKT in Wildtype cells. However, these increases were greatly suppressed in Fut8 KO HepG2 cells. Then we rescued the FUT8 gene (RES) in FUT8 KO HepG2 cells, and the reduced cell proliferation and cell signal pathways were recovered. Collectively, these results strongly suggested that core fucosylation was essential for c-Met-mediated cell proliferation. 